\title{
A New Argument for the Nomological Interpretation of the Wave Function: The Galilean Group and the Classical Limit of Nonrelativistic Quantum Mechanics
}

\author{
Valia Allori \\ Department of Philosophy \\ Northern Illinois University \\ Zulauf Hall 915, Dekalb IL 60115, USA \\ vallori@niu.edu
}

\begin{abstract}
In this paper I investigate, within the framework of realistic interpretations of the wave function in nonrelativistic quantum mechanics, the mathematical and physical nature of the wave function. I argue against the view that mathematically the wave function is a two-component scalar field on configuration space. First, I review how this view makes quantum mechanics non- Galilei invariant and yields the wrong classical limit. Moreover, I argue that interpreting the wave function as a ray, in agreement many physicists, Galilei invariance is preserved. In addition, I discuss how the wave function behaves more similarly to a gauge potential than to a field. Finally I show how this favors a nomological rather than an ontological view of the wave function.
\end{abstract}

Keywords: wave function realism, nomological interpretation of the wave function, symmetries, classical limit, Galilean group, projective rays, gauge potentials.

\section{Introduction}

Suppose that quantum mechanics can be understood as offering us a complete and realistic account of the physical world. Then, it is often claimed (Albert 2013; Lewis 2013; Ney 2013; North 2013), the fundamental physical entity which describes matter is the wave function, since it is the object whose temporal evolution quantum mechanics describes via the Schrödinger equation. This view is known with many names, including wave function ontology, or wave function realism. In fact according to this view, the wave function is regarded as a physical, material field, just like the electromagnetic field in classical electrodynamics. Consequently, the wave function is considered to be a two-component scalar field (Albert 1992), and physical space should be taken to be the space the wave function 'lives in,' namely configuration space. Differently from the case of classical electrodynamics, though, configuration space, namely the space of all the configurations of 'particles' ${ }^{1}$ in the universe, is not three-

\footnotetext{
${ }^{1}$ Here the scary quotes indicate that there are actually no particle in the universe in this approach, given that material objects are 'made of' wave functions.
} 
dimensional. Rather, if there are $N$ (three-dimensional) 'particles,' configuration space has $3 N$ dimensions.

There are a number of worries connected with this view. One is the so-called 'macro-object problem,' namely the problem of the emergence of the macroscopic threedimensional objects of our experience from the description in terms of the wave function. I will not consider this problem here, which has been analyzed extensively elsewhere (see [Allori 2013; Maudlin 2013; Monton 2013] and [Gao 2017] for critics, and [Ney forthcoming] and [Lewis 2016] for replies). Another worry is the difficulty to reconcile this view in the context of quantum field theories as pointed out in (Myrvold 2015). Instead of focusing on relativistic invariance, in this paper I discuss Galilei invariance. I show that the scalar field perspective on the wave function has undesirable consequences that can be resolved changing our view on the mathematical and physical nature of the wave function. If we think of the wave function as a projective ray, then I show that quantum mechanics preserves the Galilean symmetry, and I argue that this favors the view that the wave function has nomological character.

Here is the structure of the paper. In Section 2, I review how the view that the wave function is a scalar field makes quantum mechanics not invariant under Galilean transformations. In fact, if the wave function is a scalar field, it does not transforms in the way physics books prescribe and which makes the theory Galilei invariant. That is, if we think of the wave function as a scalar field, there is no reason for it to transform as to preserve the invariance under Galilean boosts (i.e. by multiplication of a given exponential factor), and therefore the theory is not Galilei invariant. This argument has been known for a while, and (some) proponents of this interpretation are willing to bite the bullet, arguing that we have no other choice.

In Section 3 instead I argue that there is a better way to mathematically interpret the wave function, namely as a ray in Hilbert space. This is the way many physics book already present the wave function (see, e.g. [Shankar 1994]) but hardy considered as a viable alternative in the philosophical literature. I argue instead that this is a living and preferable option.

As discussed in Section 4, it follows from the projective nature of the wave function and from the unitary evolution of the quantum state that the action of a Galilean transformation has to be described by the unitary projective representations of the Galilei group. Using the theory of representations for the Galilei group, one can show (see (Inönü and Wigner 1952)) that the only representation which has physical meaning is the so-called $m(U, s)$. This representation is the one that preserves Galilei invariance, and hence gives the right classical limit. This is not the case for the scalar field approach, which breaks Galilean symmetry and consequently does not provides the correct classical behavior under the suitable limit (see Appendix).

Furthermore, in Section 5 I discuss how if we accept the view that the wave function is mathematically a ray, then it follows that physically the wave function is 
more like a gauge potential in classical electrodynamics than a field. That is, it is determined up to a gauge transformation. Moreover, I argue that this idea supports the rival approach to wave function realism, according to which the wave function is a nomological rather than ontological entity (see [Goldstein and Zanghi 2013]).

\section{The Wave Function as a Scalar Filed and the Lack of Galilei Invariance of Quantum Mechanics}

In this section, I present the argument that if the wave function is scalar field, then quantum theory is not Galilei invariant.

Wave function realists are scientific realists, for they believe that quantum mechanics can tell us about the nature of reality, and they argue that the wave function is the mathematical object in the theory that represent physical objects. Their reasoning is straightforward, and can be summarized as follows. From the fact that the fundamental equation of Newtonian mechanics is about the behavior of points in threedimensional space we have rightly inferred that: 1) physical objects are described by these points, and 2) these points describe point-like particles. Similarly, from the fact that the fundamental equation of quantum mechanics, namely Schrödinger's equation, describes the temporal evolution of the wave function, we rightly infer that: 1) physical objects are described by the wave function, and 2) the wave function describes a material field. The reason for this is that the wave function describing a single particle is a function from vectors in three-dimensional space (the single-particle positions $r$ ) to complex numbers. If the system contains $N$ particles of positions $r_{1}, r_{2}, \ldots, r_{N}$, then the wave function will be a function from a $3 N$ dimensional space (known as configuration space) to complex numbers. Considering only this piece of information, one concludes that the wave function is a two-component scalar field. A scalar field is a field is a function whose value at each point is a scalar quantity, a real number. A familiar example of a scalar field is temperature: each location is associated with a scalar number that describes how hot (or cold) it is there. A two-component scalar field is one that is described by two scalars, which can be though as the real and the imaginary component of a complex number, or the amplitude and the phase. Albert therefore writes:

"The sorts of physical objects that wave functions are, on this way of thinking, are (plainly) fields - which is to say that they are the sorts of objects whose states one specifies by specifying the values of some set of numbers at every point in the space where they live, the sorts of objects whose states one specifies (in this case) by specifying the values of two numbers (one of which is usually referred to as an amplitude, and the other as a phase) at every point in the universe's so-called configuration space." (1996, p. 278)

A pure Galilean transformation (also known as Galilean boost) maps the description of a given system into another which differs from it only by being in constant relative motion with respect to it. Mathematically, it entails the substitution of 
$r-v t$ in place of the instances of $r$. That is: $r \rightarrow r-v t$. For instance, the description of a falling body by someone in a lab is transformed by a pure Galilean transformation into the description of the behavior of the same body as seen by someone on a train moving at constant speed with respect to the lab. A given transformation is called a symmetry if the transformation does not change the description. If so, the theory describing the phenomena is called invariant under that transformation. For instance, classical mechanics is Galilei invariant: the description of the behavior of the falling body in the lab and in the constantly moving train is the same.

Is quantum mechanics Galilei invariant? Consider a single particle system evolving freely from the point of view of the wave function realist. The wave function is a two-component scalar field $\psi$ in configuration space, which in this case is simply three-dimensional space. How will this field transform under a pure Galilean transformation? Temperature is an example of a scalar field. Think of the temperature of a desert zone. If you board a train and move away from it with constant velocity, you will not describe the desert zone as changing temperature. The description of the temperature field $T(r)$ as a function of the location after the Galilean transformation $r \rightarrow$ $r-v t$ will be $T(r) \rightarrow T(r-v t)$, and, since there is no change in temperature, we have $T(r)=T(r-v t)$. This is typically how a scalar field will change under a pure Galilean transformation: its value will stay the same. Wave function realists wants us to think of the wave function $\psi$ as a scalar field, and therefore it should transform under a Galilean transformation of magnitude $v$ as $\psi(r) \rightarrow \psi(r-v t)$. Notice that in physics book it is assumed the transformation to be like this: $\psi(r, t) \rightarrow e^{-\frac{i}{\hbar}\left[m v r+\frac{1}{2 m} v^{2} t\right]} \psi(r-v t, t)$. That is, there is an additional exponential factor that multiplies the transformed wave function. This is not compatible with thinking of the wave function as a scalar field, since there is no justification for this additional factor: it would be as if temperature would change in magnitude due to the motion.

This argument has serious consequences: if $\psi(r)$ is a solution to the free nonrelativistic single particle Schrödinger equation, then $\psi(r-v t)$ is not. That is, $\psi(r-v t)$ does not describe a possible physical state of the system. Therefore, the corresponding Schrödinger equation (constructed as an equation for the motions of a classical twocomponent scalar field) is not invariant under Galilean transformations. What would make the theory invariant is the additional exponential factor (and this is in fact the very reason why it is present in physics textbooks, as reviewed in Section 4).

This problem has been known for a while, and typically wave function realists bite the bullet and maintain that we have to learn to live with this. Look at the Schrödinger equation, they urge us. What else could it be, if not a scalar field? If so, the natural way to transform the wave function leads to Galilean symmetry breaking: as we have seen, there is no way of justifying the presence of the needed exponential factor. Instead, in the next section I argue that this is not true: I show that if the wave function 
is a ray, then it is natural for the exponential factor to be there. In this way, one recovers the Galilei invariance of the theory.

\section{The Wave Function as Ray in Projective Hilbert Space}

Remember that the conclusion that the wave function is a scalar field is mainly motivated by looking at the Schrödinger equation alone. To understand my change of perspective, notice that there are other constraints that the wave function should obey, in addition of following the Schrödinger evolution. One constraint is that wave functions which differ from a complex number should not be taken as physically distinct: $\psi$ and $c \psi$, where $c$ is a given non-zero complex number, are supposed to represent the same physical state. The reason for this is that both wave functions generate the same transition probability (Wigner 1939), (Shankar 1994). This in turns yield to a different view on the mathematical nature of the wave function. Here is the argument:

(P1) Probability distributions are proportional to the square module of the wave function;

(P2) Probability distributions make connection with experimental results;

(P3) Whatever provides the same probability distribution describes the same physical state;

(C1) Wave functions that differ by a multiplication of a complex number of unitary norm give rise to the same probability distribution;

(C2) Therefore, wave functions that differ by a multiplication of a complex number of unitary norm describe the same physical state;

(P4) Wave functions that differ by a multiplication of a complex number of unitary norm are called projective rays;

(C3) Hence, mathematically, wave functions are projective rays.

P 1 and P2 are either axioms of quantum mechanics or their consequences. P1 is Born's rule, which in case of a position measurement states that the probability of finding the particle at a given point is proportional to the square of the magnitude of the particle's wave function at that point. In general, the act of measuring a quantum system returns a number, known as the eigenvalue $a$ of the quantity being measured, which is represented by a self-adjoint operator. The probability $P$ of getting any particular eigenvalue is equal to the square of the wave function for that eigenvalue. P2 is an experimental fact since the experimental results are reproduced by the probability distributions. $\mathrm{C} 1$ simply follows mathematically if $\mathrm{P} 3$ is true: for any non-zero complex number $c, P(c \psi, \phi)=P(\psi, c \phi)=P(\psi, \phi)$. The equivalence expressed by $\mathrm{C} 1$ is mathematically implemented introducing equivalent classes. What is the best mathematical object that can express $\mathrm{C} 2$ above? The projective ray, as stated in P3. Hence, C 3 follow. In fact, wave functions can be added together and multiplied by complex numbers to form new wave functions and form a (complex) Hilbert space $H$, 
which is a vector space with inner product (necessary to compute probability distributions). The space of wave functions is therefore $H$. In order to accommodate the fact that $\psi$ and $c \psi$ represent the same physical state, a projective Hilbert state $P(H)$ of $H$ is introduced. This is the set of equivalence classes of elements of $H$ that differ by a complex number, like $\psi$ and $c \psi$. These equivalence classes are also called rays: $R \psi:=$ $\{$ for any $\phi$ in $H$ there is a complex number $c$ such that $\phi=c \psi$ \}. Any member of this set will give the same results when we use it in the Born rule, and therefore they are taken to be representing the same state. Since the wave function has to have a unit norm, to ensure probability rules to hold, it follows that $|c|^{2}=1$, thus $c=e^{i \phi}$, where $\phi$ is called global phase.

If this argument is sound it shows that the wave function is best understood as a projective object like a ray in projective Hilbert space, rather than as a scalar field. This is commonly accepted among physicists, but not among philosophers like wave function realists. Presumably, they reject P3, namely that two states that provide the same probability distribution describe the same physical state. From their point of view, this makes perfect sense. In fact, if wave functions are scalar fields, why would one think P3 is true? This would be similar to saying that a temperature field of $T=90 F$ describes the same state as the temperature field $=9,000 F$, which is clearly false: the former would presumably represent the temperature in Phoenix, AZ, during Summer, the later perhaps the temperature of the Sun. Simply rejecting P3 does not make wave function realism problematic. Nonetheless, as we saw in the previous section, this choice makes quantum theory non-Galilei invariant.

Be that as it may, in the next section I show how it follows that if the wave function is a ray then non-relativistic quantum mechanics is Galilei invariant.

\section{The Physical Representation of the Galilei Group and Classical Limit} The Galilei group is a group of transformation of space-time points that includes rotations, space and time translations, as well as pure Galilei transformations, namely constant velocity transformation. Assume that each physical system is completely described by the wave function. Group representations describe the group in terms of linear transformations. They are important because they translate group transformation in the language of linear algebra, which is well understood. In particular, they can be used to represent group elements using matrices, so that the group operations can be represented by matrix multiplication. Moreover, group representation are important from a physical perspective because they describe how a group of symmetries of a system (such as the Galilean group) affects the solutions of the equations describing the system. According to a theorem (Wigner 1939), any symmetry in quantum mechanics can be represented by a unitary operator acting in the space of physical states. Since the wave function is a ray, it is a projective object, and as such we have to look at the action of the so-called projective representation of the Galilean group. Moreover, because the 
wave function evolution yields a unitary evolution, one has to restrict to the action of the projective and unitary representations of the Galilei group (Inönü and Wigner 1952), (Bargmann 1954), (Levy-Leblond 1963; 1971). In general, to any invariance group in quantum mechanics should correspond a projective unitary representation of the group. In the case of the Galilean group, there are several such representations which are candidates to represent the way in which the wave function is affected by a transformation in the Galilei group. Inönü and Wigner, as well as Levy-Leblond, have argued that the only projective unitary representation which can be interpreted physically is the one dubbed $m(U, s)$. They reason is that a physical representation should fulfill additional physical requirements, such as the existence of a position operator. That is, they demand that there exists an (Hermitian) operator $\boldsymbol{X}$ transforming correctly under roto-translations, namely $\boldsymbol{X} \rightarrow R(\boldsymbol{X})+\boldsymbol{a}$, with $R$ a rotation and $\boldsymbol{a}$ a space translation. It can be shown that such an operator can only be constructed within $m(U, s)$. In this case the position operator is $\boldsymbol{X}=\frac{1}{m} \boldsymbol{K}$ that operates on wave functions on momentum space, or $\boldsymbol{X}=\nabla_{\boldsymbol{r}}$ acting in configuration space. Because of this, $m(U, s)$ should be considered the only physical representation of the Galilean group in quantum mechanics. This representation can be interpreted as describing particles with mass $m$, internal energy $U$ and spin $s$ (see again [Levy-Leblond 1971]). Writing the representation $m(U, s)$ in configuration space and in the Schrödinger picture, one has: $\psi_{\mu}(\boldsymbol{r}, t)=\int d^{3} p e^{-i\left(U+\frac{p^{2}}{2 m}\right)-i \boldsymbol{p} \cdot \boldsymbol{r}} \psi_{\mu}(\boldsymbol{p})$. From the properties of $m(U, s)$ one can find that a pure Galilei transformation maps the original wave function as follows: $\psi(x, t) \rightarrow$ $e^{-\frac{i}{\hbar}\left[m v x+\frac{m}{2} v^{2} t\right]} \psi(x-v t, t)$, which is the transformation we find in physics textbooks that wave function realists rejected.

The bottom line is therefore that if the wave function is a ray, in order to see how the Galilei group acts on it, one would look at the projective unitary representations of such group. In doing so, it has been shown that the only such transformation with physical significance is the one that correspond to a transformation of the wave function that makes quantum mechanics relativistic invariant. In contrast, and not surprisingly, the transformation of the wave function that the wave function realists propose is not among the projective unitary representations of the Galilei group. This would correspond to represent the group with the identity transformation, which means, for a pure Galilei transformation in one dimension, $\psi(x, t) \rightarrow \psi(x-v t, t)$. Obviously, there is nothing intrinsically mistaken with this transformation. However, as we already have seen, it makes quantum theory non-Galilei invariant. As already noted, wave function realists often bite the bullet, claiming that there is no alternative choice of the mathematical nature of the wave function that would explain the need of the transformation needed to preserve Galilei invariance. In contrast, I have presented such alternative in the previous section, considering the wave function as a projective object. 
This choice preserves the symmetry and, in contrast to what claimed by wave function realists, it explains why the wave function transforms as to preserve Galilean symmetry.

One could still insist that symmetries are not a big deal. Nonetheless, it is worth underlining that since classical mechanics is Galilean invariant a non-Galilei invariant quantum mechanics would give rise to the wrong classical limit. I show this in the appendix for a free particle in one dimension. I think this is a serious issue, since it makes difficult to see how one could recover classical mechanics from quantum theory. This perhaps will make the unwelcomed consequences of the lack of Galilean invariance more apparent.

\section{The Wave Function as a Gauge-Type Potential and a New Argument for the Nomolgical Interpretation of the Wave Function}

In this section I argue that a consequence of my proposed view is that the wave function behaves more like a gauge potential than like a field (scalar or not). Then, I use this result to construct a new argument for the nomological view of the wave function.

Let me start with first point. To make an analogy with electromagnetism, the claim is that wave function looks more like $A$ and $V$, the magnetic and electric potentials in classical electrodynamics, than the electromagnetic fields $E$ and $B$. The traditional way of looking at classical electrodynamics is that the electric and magnetic fields are the ones describing the physical degrees of freedom, rather than the potentials. There are certain transformations of the potentials, called gauge transformations, which do not affect the observed electric and magnetic fields: $V \rightarrow V+\frac{\partial \lambda}{\partial t} ; A \rightarrow A-\nabla \lambda$, where $\lambda$ is a scalar function. Since the fields are not affected by this gauge transformations and they are supposed to capture the physical description of electrodynamics, electrodynamics is said to be gauge invariant and the potentials are dubbed gauge potentials. Once one choses a gauge, the gauge potentials are fixed. Clever choices of $\lambda$ will simplify one of the other of the differential equations for the potentials. The solution of these simpler equations for the transformed potentials gives the same fields as the solutions to the untransformed, complicated equations. For instance, the Coulomb gauge is the one in which one chooses a curl-free magnetic potential and simplifies the equation for the electric potential $V$, but it's hard to compute the magnetic potential in this gauge. On the other hand, the Lorentz gauge is more useful in timedependent cases. Be that as it may, the point is that the choice of the gauge is made as a matter of computational convenience. This gauge freedom is directly connected to the fact that gauge potentials are seen as equivalent classes that differ by a gauge transformation. Similarly, if the wave function is a ray, that is a set of equivalent classes up to a phase, one would have something similar to gauge freedom also in quantum mechanics. As in classical electrodynamics one fixes the gauge as a matter of convenience, the same can be done in quantum mechanics as well. 
The phase $c$ between $\psi$ and $c \psi$ can depend on time, and as such can be seen as a time gauge: $c=c(t)$. If so, Schrödinger's equation is the result of fixing the gauge in a particularly convenient way. That is, choosing $\left|\frac{d}{d t} c(t)\right|=0$, the evolution for the wave function becomes the Schrödinger equation, which has the convenient features of being deterministic, unitary and linear².

If one accepts the argument above then I argue that it also follows that the wave function is best seen as a nomological entity rather than an object which represents matter. One may write an argument by analogy:

$\left(\mathrm{P}^{\prime} 1\right)$ if the wave function is a projective object then it is similar to the gauge potentials in electromagnetism (from the argument above);

$\left(\mathrm{P}^{\prime} 2\right)$ the gauge potentials in classical electrodynamics are law-like (nomological);

(C) Therefore the wave function is law-like (nomological).

Let us discuss the second premise $\mathrm{P}^{\prime} 2$. One should point out that the reason why the electromagnetic potentials are not considered as representing matter is that, in contrast to the fields, they are fixed up to a gauge. That is, they are equivalent classes, rather than single objects. Arguably because of this reason, the most accepted view is that classical electrodynamics is about the behavior of particles and fields, not gauge potentials. So what is the role of the gauge potential in classical electrodynamics? Why do they exist in the theory? The common answer is that they 'generate the fields.' It seems to me that the safest way to interpret this, in light of the fact that they are not considered as representing something material, is that they are part of the ingredients to generate the law of evolution of the electromagnetic fields:

$$
\boldsymbol{E}=-\nabla V-\frac{\partial \boldsymbol{A}}{\partial t} ; \boldsymbol{B}=\nabla \times \boldsymbol{A}
$$

In this sense, they are part of the law and as such have a nomological character ${ }^{3}$.

In orthodox quantum mechanics the Schrödinger evolving wave function provides the complete description of the system. We are in the assumption that the wave function is mathematically a ray. Now the question becomes: Is a ray a suitable mathematical object to represent physical things? The discussion above from electromagnetism suggests that it is not: rays behave like gauge potentials, and things

\footnotetext{
${ }^{2}$ One relevant difference between quantum mechanics and electrodynamics is that while in classical electrodynamics Maxwell's equations of motions (in addition to Newton's law) are for the electromagnetic fields $E$ and $B$ and not in terms of their gauge potentials, in quantum mechanics just the opposite is true. The fundamental equation (the only one there is) is in terms of the gauge field $\psi$. While in classical electrodynamics the invariant quantities are the fields, the gauge invariant objects of quantum mechanics are the transition probabilities, which are defined in term of the wave function.

${ }^{3}$ The situation is actually more complicated, since in classical electrodynamics we have particles, which are affected by fields, which are affected by potentials. Because of this one may also think that the fields are law-like too, in addition to the potentials: they are part of the ingredients that are necessary to determine the evolution of the particles (See (Allori 2015) for a discussion of this.).
} 
that are defined up to a gauge do not seem to be good candidates to represent matter. If so, the wave function, being a ray, is not the right kind of object to represent physical objects. One could question this, but then she will have to either accept that gauge potential are material in the case of electrodynamics as well, or show that there is an important disanalogy.

We ended up to the (usual) question: if the wave function does not represent matter, then what is it? The gauge potential analogy suggests the answer: the wave function is nomological. In fact, the wave function is one of the ingredients to generate the law of evolution of something else: in the case of orthodox quantum theory, presumably the probability distribution. As suggested by Allori et al (2008), in orthodox quantum mechanics the probability distributions are more primitive than the wave function, and it should be taken as the mathematical entity in the theory that represent matter. In the terminology of the primitive ontology approach, they are the primitive ontology of orthodox quantum mechanics. In contrast, the wave function is the type of mathematical object in the theory that, just like gauge potential in electromagnetism, generates the law for the evolution of matter. This is the sense in which the wave function contributes to quantum mechanics without representing material entities.

\section{Acknowledgements}

This paper goes back a long way, so I simply wish to thank everyone who has ever discussed symmetry properties in connection with primitive ontology. Also, I am very thankful to two anonymous referees whose comments highly contributed to make this version of the paper better than the original. 


\section{APPENDIX: The Classical Limit of Quantum Mechanics for a Free Gaussian Wave Packet for the Wave Function as a Field and as a Ray}

Consider a free particle on a line described by a wave packet, as is usual in ordinary quantum mechanics. To simplify calculations, consider the case of a (one-dimensional) Gaussian wave packet centered in $x_{0}=0$ at time $t=0$ :

$$
\psi(x, 0)=\frac{1}{\left(2 \pi \sigma_{0}\right)^{1 / 4}} e^{-\frac{x^{2}}{4 \sigma_{0}}+i \frac{m u}{\hbar x}} .
$$

In the expression, $\sigma_{0}$ is the initial spreading of the packet and $u$ is the velocity. At time $t$ the packet has evolved to the form

We can write it as

$$
\psi(x, t)=\frac{1}{\left[\sqrt{2 \pi \sigma_{0}}\left(1+\frac{i \hbar t}{2 m \sigma_{0}^{2}}\right)\right]^{1 / 2}} e^{-\frac{(x-u)^{2}}{4 \sigma_{0}^{2}\left(1+\frac{i \hbar t}{2 m \sigma_{0}^{2}}\right)}+i \frac{m u}{\hbar}\left(x-\frac{u}{2} t\right)} .
$$

where $S$ is given by

$$
\psi(x, t)=R(x, t) e^{\frac{i}{\hbar} S(x, t)},
$$

$$
S(x, t)=m u\left(x-\frac{u}{2} t\right)+\frac{1}{2}\left(\frac{\hbar t}{2 m \sigma_{0}^{2}}\right)^{2} \frac{(x-u t)^{2} \frac{m}{t}}{1+\left(\frac{\hbar t}{2 m \sigma_{0}^{2}}\right)^{2}}
$$

and $R$ by

$$
R(x, t)=\frac{1}{\left[\sqrt{2 \pi \sigma_{0}}\left(1+\frac{i \hbar t}{2 m \sigma_{0}^{2}}\right)\right]^{\frac{1}{2}}} e^{-\frac{(\mathrm{x}-\mathrm{ut})^{2}}{4 m \sigma_{0}^{2}\left(1+\left(\frac{\hbar t}{2 m \sigma_{0}^{2}}\right)^{2}\right)} .}
$$

According the general formulation of the classical limit in the framework of orthodox quantum mechanics [Maslov 1981], the classical velocity associated to the particle is given by $V=\frac{\nabla S_{0}}{m}$, where $S_{0}$ is obtained by $S$ in the limit ' $\hbar \rightarrow 0^{\prime} .{ }^{4}$ In the case of a free Gaussian wave packet, we have $S_{0}=m u x-\frac{1}{2} m u^{2} t+O\left(\hbar^{2}\right)$, and therefore $V=u$. If this is the velocity of the particle associated to the packet in the classical limit, one should expect that it would transform under a pure Galilean transformation (in one spatial dimension) of velocity $v$ corresponding to $V \rightarrow V-v$, since classical mechanics is Galilei invariant. This is not the case if the wave function transforms according to the scalar field transformation as proposed by the wave function realists. In fact, supposing that under the transformation $x \rightarrow x-v t$, the wave function transforms as $\psi(x, t) \rightarrow$ $\psi(x-v t)$. Then, the action $S$ will transform as $S(x, t) \rightarrow S(x-v t, t)$ where $S(x-v t)$ is

\footnotetext{
${ }^{4}$ This limit should be intended as the mathematical implementation of considering a situation in which the relevant action of the system is big compared to $\hbar$.
} 


$$
S(x-v t, t)=m u x-\frac{1}{2} m u^{2} t+\frac{1}{2}\left(\frac{\hbar t}{2 m \sigma_{0}^{2}}\right)^{2} \frac{[x-(u+v)]^{2} \frac{m}{t}}{1+\left(\frac{\hbar t}{2 m \sigma_{0}^{2}}\right)^{2}} .
$$

In the classical limit it becomes (up to a factor $O\left(\hbar^{2}\right)$ )

$$
S_{0}(x-v t, t)=m u\left[x-\left(\frac{u}{2}+v\right) t\right],
$$

that is , $V=u$, which is not the expected result $u-v$.

As expected, the correct limit is instead obtained if the wave function transforms under a pure one dimensional Galilei transformation according to the representation $m(U, s)$ in configuration space. That is, we have

$$
\psi(x, t) \rightarrow e^{-\frac{i}{\hbar}\left[m v x+\frac{1}{2} m v^{2} t\right]} \psi(x-v t, t) .
$$

We can see that with that transformation the correct classical limit is now obtained. In fact the action $S$ transforms as

$$
S(x, t) \rightarrow m(u-v) x-\frac{m}{2}\left(u^{2}+v^{2}\right) t+\frac{1}{2}\left(\frac{\hbar t}{2 m \sigma_{0}^{2}}\right)^{2} \frac{\left[x-(u+v)^{2} \frac{m}{t}\right.}{1+\left(\frac{\hbar t}{2 m \sigma_{0}^{2}}\right)^{2}}
$$

that gives in the classical limit (up to a factor $O\left(\hbar^{2}\right)$ )

$$
S^{0}(x, t) \rightarrow m(u-v) x-\frac{m}{2}\left(u^{2}+v^{2}\right) t
$$

and therefore we get $V=u-v$, as expected. 


\section{References}

Albert, David Z. 1992. "Experimental Quantum Metaphysics." In Bohmian Mechanics and Quantum Theory: An Appraisal, edited by J. Cushing, A. Fine and S. Goldstein, 277-287. Dordrecht: Kluwer.

Albert, David Z. 2000. Time and Chance. Cambridge: Harvard University Press.

Albert, David Z. 2013. "Wave Function Realism." In The Wave Function: Essays in the Metaphysics of Quantum Mechanics, edited by D.Z. Albert and A. Ney, 52-57. New York: Oxford University Press.

Albert, David Z. and Alyssa Ney, eds. 2013. The Wave Function: Essays in the Metaphysics of Quantum Mechanics. New York: Oxford University Press.

Allori, Valia. 2013. "Primitive Ontology and the Structure of Fundamental Physical Theories." In The Wave Function: Essays in the Metaphysics of Quantum Mechanics, edited by D.Z. Albert and A. Ney, 58-75. New York: Oxford University Press.

Allori, Valia. 2015. "Maxwell's Paradox: Classical Electrodynamics and its Time-reversal Invariance." Analytica 1:1-19.

Allori, Valia, Sheldon Goldstein, Roderich Tumulka and Nino Zanghí. 2008. "On the Common Structure of Bohmian Mechanics and the Ghirardi-Rimini-Weber Theory." The British Journal for the Philosophy of Science 59 (3): 353-389.

Arntzenius, Frank. 2004. “Time-reversal Operation, Representation of the Lorentz Group and the Direction of Time." Studies in Histories and Philosophy of Modern Physics 35 (1): 31-43.

Bargmann, Valentine. 1954. “On Unitary Ray Representations of Continuous Group.” Annals of Mathematics 59 (1): 1-46.

Earman, John. 2002. "What Time-reversal Invariance is and Why it Matters." International Studies in the Philosophy of Science 16: 245-264.

Gao, Shan. 2017. The Meaning of the Wave Function: In Search of the Ontology of Quantum Mechanic. Cambridge: Cambridge University Press.

Inönü, Erdal and Eugene P. Wigner. 1952. "Representations of the Galilei Group." Il Nuovo Cimento IX (8): 708-718. 
Levy-Leblond, Jean-Marc. 1963. “Galilei Group and Nonrelativistic Quantum Mechanics." Journal of Mathematical Physics 4, 776-788.

Levy-Leblond, Jean-Marc. 1971. "Galilei Group and Galilean Invariance.” In Group Theory and its Applications Vol. 2, edited by E. M. Loebl, 221-299. New York: Academic Press.

Lewis, Peter J. 2015. "In Search of Local Beables." International Journal of Quantum Foundations 1: 215-229.

Lewis, Peter J. 2013."Dimensions as illusions." In The Wave Function: Essays in the Metaphysics of Quantum Mechanics, edited by D.Z. Albert and A. Ney, 110-125. New York: Oxford University Press.

Ney, Alyssa. Forthcoming. "Finding the World in the Wave Function: Some Strategies for Solving the Macro-object Problem." Synthese.

Ney, Alyssa. 2013. "Ontological Reduction and the Wave Function Ontology." In The Wave Function: Essays in the Metaphysics of Quantum Mechanics, edited by D.Z. Albert and A. Ney, 168-183. New York: Oxford University Press.

Malament, David. 2004. "On the Time-reversal Invariance of Classical Electromagnetic Theory." Studies in Histories and Philosophy of Modern Physics 35 B: 295-315.

Maslov, Victor P. and M. V. Fedoriuk. 1981. “Semi-Classical Approximation in Quantum Mechanics. “Boston: Reidel.

Monton, Bradley. 2013. "Against 3N-Dimensional Space." In The Wave Function: Essays in the Metaphysics of Quantum Mechanics, edited by D.Z. Albert and A. Ney, 154-167. New York: Oxford University Press.

Myrvold, Wayne. 2015. “What is a Wave Function?” Synthese 192 (10): 3247-3274.

North, Jill. 2013. "The Structure of the Quantum World." In The Wave Function: Essays in the Metaphysics of Quantum Mechanics, edited by D.Z. Albert and A. Ney, 184-202. New York: Oxford University Press.

North, Jill. 2008. “Two Views of Time-reversal." Philosophy of Science 75 (2): 201-223. 
Shankar, Ramamurti. 1994. Principles of Quantum Mechanics. New York:Plenum Press.

Wigner, Eugene P. 1939. “On the Unitary Representations of the Inhomogeneous Lorentz Group." Annals of Mathematics 40: 149-204. 